\title{
Perioperative comparison of the agreement between a portable fingertip pulse oximeter $v$. a conventional bedside pulse oximeter in adult patients (COMFORT trial)
}

\author{
R N Smith, MB ChB, DA (SA), FCA (SA), MMed (Anaes); R Hofmeyr, MB ChB, Dip PEC (SA), DA (SA), FCA (SA), MMed (Anaes), FAWM \\ Department of Anaesthesia and Perioperative Medicine, Groote Schuur Hospital and Faculty of Health Sciences, University of Cape Town, \\ South Africa
}

Corresponding author: $R$ N Smith (reubensmith@hotmail.co.za)

\begin{abstract}
Background. Low-cost, portable fingertip pulse oximeters are widely available to health professionals and the public. They are often not tested to International Organization for Standardization standards, or only undergo accuracy studies in healthy volunteers under ideal laboratory conditions.

Objectives. To pragmatically evaluate the agreement between one such device and a conventional bedside pulse oximeter in a clinical setting, in patients with varied comorbidities and skin pigmentations.

Methods. A single-centre equipment comparison study was conducted. Simultaneous measurements were obtained in 220 patients with both a Contec CMS50D Fingertip Pulse Oximeter and a Nihon Kohden Life Scope MU-631 RK conventional bedside monitor. Peripheral oxygen saturations $\left(\mathrm{SpO}_{2}\right)$ and pulse rates were documented, and patients' skin tone was recorded using the Fitzpatrick scale. Data were assessed using a Bland-Altman analysis with bias, precision and limits of agreement (LOA) calculated with 95\% confidence intervals (CIs) A priori acceptability for LOA was determined to be $3 \%$, in keeping with international standards.

Results. The mean difference (therefore bias) between the conventional and fingertip oximeters for all data was $-0.55 \%$ ( $95 \%$ CI $-0.73-$ -0.36). Upper and lower limits of agreement were 2.16\% (95\% CI $1.84-2.47$ ) and -3.25\% (95\% CI -3.56 - -2.94). Regression analysis demonstrated worsening agreement with decreasing $\mathrm{SpO}_{2}$. When samples were separated into 'normal' (SpO $\left.293 \%\right)$ and 'hypoxaemic' $\left(\mathrm{SpO}_{2}<93 \%\right)$ groups, the normal range displayed acceptable agreement between the two oximeters (bias $-0.20 \%$ with LOA 2.20 - -2.27 ), while the hypoxaemic group fell outside the study's a priori limits. Heart rate measurements had a mean difference of -0.43 bpm (LOA -5.61 - 4.76). The study was not powered to detect differences among the skin tones, but demonstrated no trend for this parameter to alter the $\mathrm{SpO}_{2}$ measurements.

Conclusions. During normoxia, portable fingertip pulse oximeters are reliable indicators of $\mathrm{SpO}_{2}$ and pulse rates in patients with various comorbidities in a pragmatic clinical context. However, they display worsening agreement with conventional pulse oximeters during hypoxaemia. Skin tones do not appear to affect measurements adversely.
\end{abstract}

S Afr Med J 2019;109(3):154-158. DOI:10.7196/SAMJ.2019.v109i3.13633

The ability to assess a patient's arterial oxygen saturation accurately and non-invasively has become an accepted standard of care in the perioperative period. ${ }^{[1]}$ Pulse oximetry is considered an essential item of equipment for providing safe anaesthesia by various regulatory bodies throughout the world, including the South African Society of Anaesthesiologists and the World Federation of Societies of Anaesthesiologists, ${ }^{[2,3]}$ which adopted the International Standards for a Safe Practice of Anaesthesia ${ }^{[4]}$ in 1992. Pulse oximeters are included in the World Health Organization (WHO)'s Surgical Safety Checklist (SSC), ${ }^{[5]}$ which is used in health facilities throughout the world before commencement of a surgical procedure. Studies have demonstrated that the WHO SSC has nearly doubled patients' likelihood of receiving proven standards of surgical care, and substantially reduced complications and deaths. ${ }^{[6]}$

Pulse oximetry is non-invasive, safe and currently performed routinely on all surgical patients during admission, intraoperatively and postoperatively, without requiring specific consent. It is more cost-effective, less painful, easier to perform and more readily available than arterial blood gas analysis. It has largely replaced this method in many clinical situations, unless carbon dioxide or acid-base status is specifically required. Portable fingertip pulse oximeters have the additional benefits of being cost-effective, highly compact, portable, battery operated and easy to use. When pulse oximeters are manufactured, they are tested for accuracy against blood gas analysis in healthy volunteers breathing hypoxic gas mixtures under ideal laboratory conditions. There are published standards from the US Food and Drug Administration (FDA) on how this should be performed, including statistical methods appropriate for device comparison, such as Bland-Altman analysis. ${ }^{[7]} \mathrm{A}$ review of the recent literature reveals a paucity of accuracy studies utilising fingertip pulse oximeters in patients in the clinical setting.

\section{Objectives}

To pragmatically investigate the performance of these devices in adult surgical patients in a hospital/theatre environment. Studying the performance of portable fingertip pulse oximeters may result in the availability of cheaper devices in medical facilities in lowto middle-income areas, give anaesthetists and other medical personnel the confidence to make clinical decisions based on these highly portable devices, add further data to existing studies, and help assess whether darker skin pigmentation affects the performance of these devices in the clinical setting, which is highly relevant in an African country such as South Africa (SA). 


\section{Methods \\ Patients}

This prospective, quantitative equipment comparison study took place at Groote Schuur Hospital, a tertiary-level institution in Cape Town, SA, over a 4-week period. Institutional approval was granted by the University of Cape Town's Human Research Ethics Committee (ref. no. HREC 572/2017). Written informed consent was waived on the provision that no patient-identifiable data were collected and pulse oximetry was already being used for routine monitoring purposes. Furthermore, simple verbal consent for inclusion was obtained from conscious patients. All adult surgical patients aged $\geq 18$ years who presented for elective or emergency surgery were considered eligible for the study and recruited by convenience sampling in perioperative areas such as pre-assessment clinics, recovery rooms, operating theatres and intensive care units (ICUs). Exclusion criteria (Table 1) were conditions known to cause inaccuracies in pulse oximetry, or infectious diseases with a high risk of transmission. ${ }^{[8-14]}$

\section{Measurements}

Data were recorded by the principal author (RNS) on an Excel spreadsheet, version 16.19 (Microsoft, USA) and archived in password-protected Cloud storage. The CMS50D Fingertip Pulse Oximeter (Contec, USA) was selected as the test device for this study because of its relatively low cost ( ZAR500, compared with the control bedside pulse oximeter, which costs $\sim$ ZAR200 000), its ease of availability in SA, and the fact that that it was one of two devices identified that met International Organization for Standardization (ISO) and FDA standards in healthy test subjects in a prior study. ${ }^{[15]}$ The device was purchased privately by the authors. A Life Scope MU-631 RK bedside monitor (Nihon Kohden, Japan) was provided by the Department of Anaesthesia and Perioperative Medicine at Groote Schuur Hospital for the control measurements. It is a commonly used monitor in the hospital and was calibrated by the manufacturer.

Sample size was calculated using results obtained from an unpublished pilot study performed using the same methods. The Monte-Carlo simulation method, as outlined by Lu et al., ${ }^{[16]}$ was used, utilising MedCalc Statistical Software version 18.6 (MedCalc, Belgium; http://www.medcalc.org). A maximum difference in peripheral oxygen saturation $\left(\mathrm{SpO}_{2}\right)$ of $3 \%$, type I error 0.05 and type II error 0.01 gave a sample size estimate of 220 .

The fingertip and conventional bedside pulse oximeter probes were applied simultaneously to the same hand, contralateral to the blood pressure cuff. Once the waveform on both pulse oximeters was confirmed, readings were taken at 30 seconds post-application to allow for the time averaging of each pulse oximeter. Non-patient identifiable data were recorded, limited to $\mathrm{SpO}_{2}$, heart rate, skin colour (estimated by the Fitzpatrick scale) and qualitative waveform strength of signal (good or poor). Similar to other studies, the Fitzpatrick scale was selected (rather than methods such as the von Luschan scale or reflectance spectrophotometry) for reasons of simplicity, repeatability and cost containment. ${ }^{[15,17-19]}$ There was no interruption to existing patient monitors. The study devices were cleaned with a commercial antiseptic solution between patients.

\section{Statistical analysis}

Bland and Altman have established bias and precision estimates as the standard reported statistic when comparing agreement between a new or less-established measurement technique and an established one. ${ }^{[20-23]}$ When the a priori limits of agreement are small (95\% limits of agreement defined as bias plus or minus 1.96 standard deviations)

\section{What questions this study addressed}

This pragmatic, prospective study compared the agreement between the measured arterial oxygen saturation levels and pulse rates using a portable fingertip v. a conventional bedside pulse oximeter in adult patients presenting for elective and emergency surgery, in a clinical setting.

\section{What this study adds to our knowledge}

This study found that there is sufficient agreement between the arterial oxygen saturations and pulse rates measured by a portable fingertip and conventional bedside pulse oximeters among adult surgical patients who are not hypoxaemic in the clinical setting. However, as saturations dropped into the hypoxaemic range ( $<93 \%)$, agreement between the devices worsened. Additionally, it found that skin tone had no significant influence on the measurements, although the study was not powered to detect this outcome convincingly.

\section{How this study could change clinical practice}

Low-cost fingertip pulse oximeters marketed for non-medical use are often not tested in humans under clinical conditions. The measurements obtained with these portable and affordable items of equipment have therefore been regarded with scepticism by medical staff. This study demonstrates that one such pulse oximeter is as accurate as a far more expensive bedside pulse oximeter when used to exclude hypoxia. Similar future studies can be done using other portable fingertip pulse oximeters to promote access to these vital medical instruments in resource-limited areas.

\section{Table 1. Exclusion criteria}

\section{Significantly low blood pressure or cold peripheries}

Excessive patient movement

Abnormal haemoglobin variants

Certain dyes used during the operation (e.g. methylene blue)

Nail polish/black henna

Bandages/tape, etc. on the hand/finger

Inadequate pulse oximeter trace

and not of clinical importance, the two measurement techniques can be considered interchangeable. For this study, we determined that an $\mathrm{SpO}_{2}$ difference of $<3 \%$ is not of clinical importance, which is in keeping with the ISO and FDA testing protocol. Data analysis was performed by the authors with Medcalc (as above).

\section{Results}

We obtained 220 simultaneous pulse oximetry measurements. $\mathrm{SpO}_{2}$, pulse rate, skin-tone classification and the clinical setting are summarised in Table 2. The mean difference (bias) between the conventional and fingertip oximeters for all data was $-0.55 \%$ ( $95 \%$ confidence interval (CI) $-0.73--0.36$, Fig. 1). Upper and lower limits of agreement were $2.16 \%$ (95\% CI $1.84-2.47)$ and $-3.25 \%(-3.56-$ -2.94). Regression analysis demonstrated worsening agreement with decreasing $\mathrm{SpO}_{2}$. When samples were separated into 'normal' $\left(\mathrm{SpO}_{2} \geq 93 \%\right.$, Fig. 2$)$ and 'hypoxaemic' $\left(\mathrm{SpO}_{2}<93 \%\right.$, Fig. 3) groups, the normal range displayed acceptable agreement between the two oximeters (bias $-0.20 \%$ with limits of agreement (LOA) $2.20--2.27$ ), while the hypoxaemic group fell outside the study's a priori limits. Heart rate measurements had a mean difference of $-0.43 \mathrm{bpm}$ (LOA $-5.61-4.76$, Fig. 4). The study was not powered to detect difference among the skin tones, but demonstrated no trend for this parameter to alter the $\mathrm{SpO}_{2}$ measurements (Fig. 5). 


\begin{tabular}{|c|c|c|c|}
\hline & All & $\mathrm{SpO}_{2} \geq 93 \%$ (normal) & $\mathrm{SpO}_{2}<93 \%$ (hypoxaemic) \\
\hline \multicolumn{4}{|l|}{$\mathrm{SpO}_{2}, \%(95 \% \mathrm{CI})$} \\
\hline$n$ & 220 & 164 & 56 \\
\hline Mean difference (bias) & $-0.55(-0.73--0.36)$ & $-0.20(-0.38--0.01)$ & $-1.57(-1.92--1.22)$ \\
\hline Lower LOA & $-3.25(-3.56--2.94)$ & $-2.60(-2.92--2.27)$ & $-4.13(-4.73--3.53)$ \\
\hline Upper LOA & $2.16(1.84-2.47)$ & $2.20(1.88-2.53)$ & $0.99(0.37-1.59)$ \\
\hline Pulse rate, \% (95\% CI) & & - & - \\
\hline$n$ & 220 & & \\
\hline Mean difference (bias) & $-0.43(-0.78--0.08)$ & & \\
\hline Lower LOA & $-5.61(-6.22--5.01)$ & & \\
\hline Upper LOA & $4.76(4.16-5.37)$ & & \\
\hline Skin tone (Fitzpatrick scale), $n$ (\%) & & - & - \\
\hline I (pale white skin, e.g. British) & $12(5.5)$ & & \\
\hline II (white skin, e.g. Scandinavian) & $28(12.7)$ & & \\
\hline III (light brown skin, e.g. Central European) & $69(31.4)$ & & \\
\hline IV (moderate brown/olive skin, e.g. Asian, Latino) & $45(20.5)$ & & \\
\hline V (dark brown skin, e.g. Native American) & $28(12.7)$ & & \\
\hline VI (very dark/black skin, e.g. African) & $38(17.3)$ & & \\
\hline Location, $n(\%)$ & & - & - \\
\hline ICU & $60(27.3)$ & & \\
\hline Recovery room & $110(50.0)$ & & \\
\hline Respiratory clinic & $31(14.1)$ & & \\
\hline Theatre & $19(8.6)$ & & \\
\hline
\end{tabular}

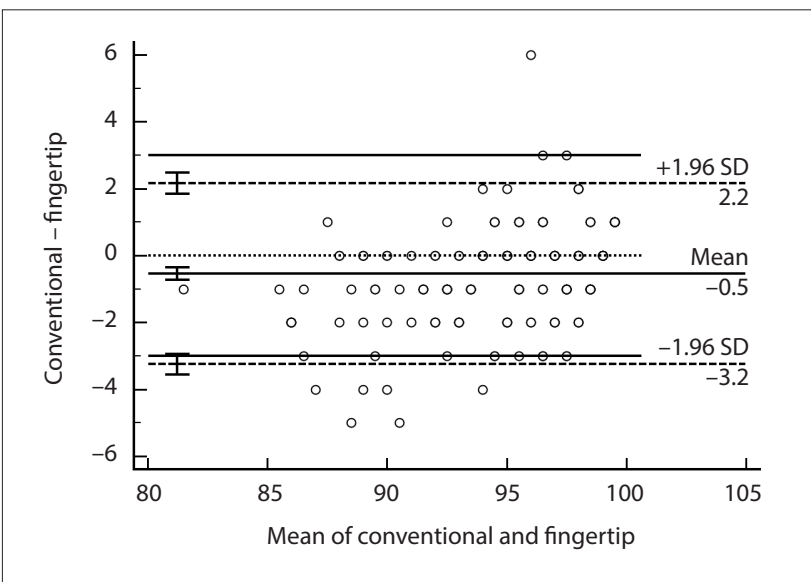

Fig. 1. Bland-Altman plot for all peripheral oxygen saturation measurements (\%). $(S D=$ standard deviation. $)$

\section{Discussion}

Pulse oximeters non-invasively measure and display heart rate and $\mathrm{SpO}_{2}$ derived from photoplethysmographic measurements at two rapidly alternating wavelengths of light. ${ }^{[10-12,24]}$ Oxygenated blood (oxyhaemoglobin) and deoxygenated blood (deoxyhaemoglobin) differ in their absorption spectra. A sensor containing a light-emitting diode and a light-detecting photodiode are placed on a cutaneous vascular bed, such as a fingertip or earlobe, that can be transilluminated. Arterial pulsations are identified by plethysmography, allowing corrections for light absorption by non-pulsating venous blood and tissue. The ratio of absorption at the red and infrared wavelengths is analysed by a microprocessor, and $\mathrm{SpO}_{2}$ is estimated from a stored calibration curve.

Oximeter testing is based on experimental measurements in healthy volunteers who are subjected to rebreathing controlled

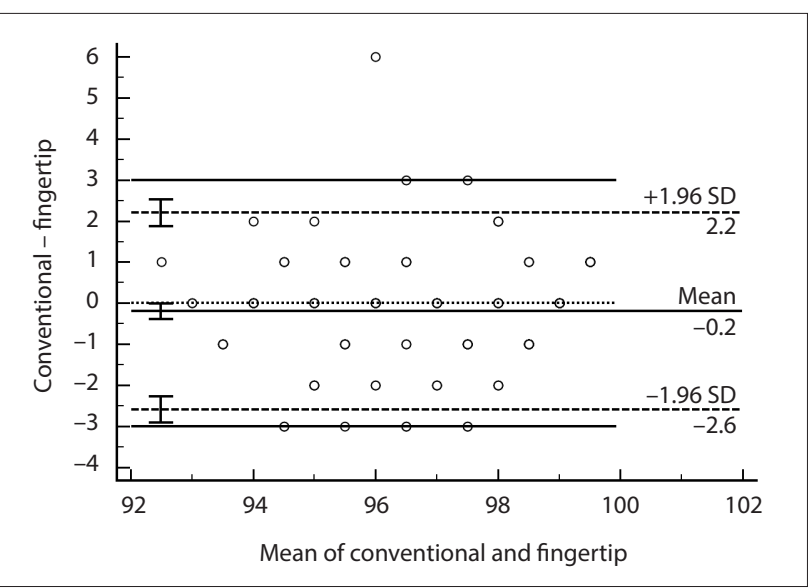

Fig. 2. Bland-Altman plot for peripheral oxygen saturation measurements $\geq 93 \%$ (\%). (SD = standard deviation.)

hypoxic gas mixtures under laboratory conditions, which includes controlling for subject movement and ambient light. Oxygen saturation of haemoglobin is then determined by both the pulse oximeter $\left(\mathrm{SpO}_{2}\right)$ and in vitro laboratory multiwavelength co-oximeter $\left(\mathrm{SaO}_{2}\right.$, considered the gold standard). Pulse oximeters are inaccurate at low blood oxygen saturations because researchers are limited in terms of the degree of hypoxaemia inducible in volunteers. The FDA requires pulse oximeters marketed for medical use to be tested in the $\mathrm{SaO}_{2}$ range $70-100 \%$. The shape of the calibration curves is extrapolated at oxygen saturations below these levels. Each manufacturer's calibration curve is proprietary.

Numerous studies have confirmed the accuracy of conventional pulse oximeters when compared with co-oximeters in the clinically relevant range of arterial oxygen saturation. ${ }^{[13,25-27]}$ Accuracy 


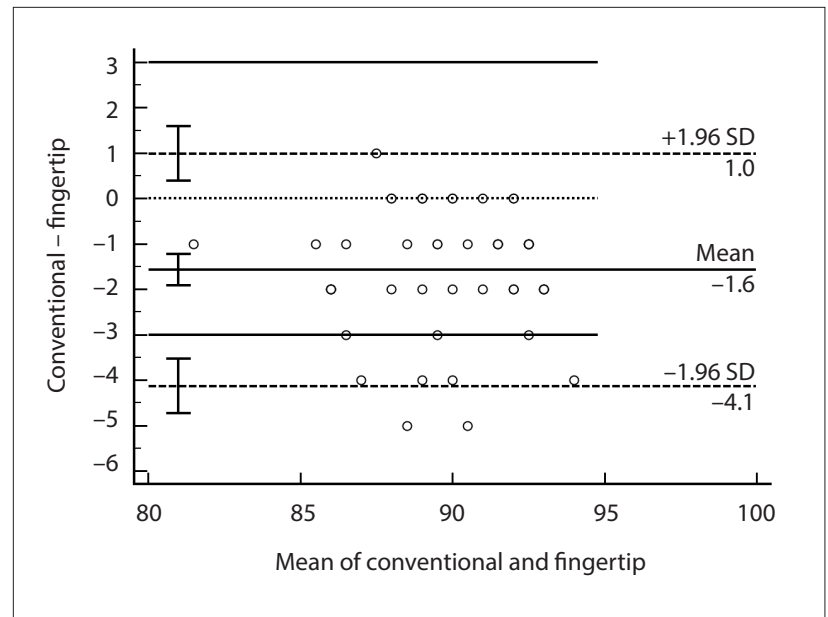

Fig. 3. Bland-Altman plot for peripheral oxygen saturation measurements $<93 \%$ (\%). (SD = standard deviation.)

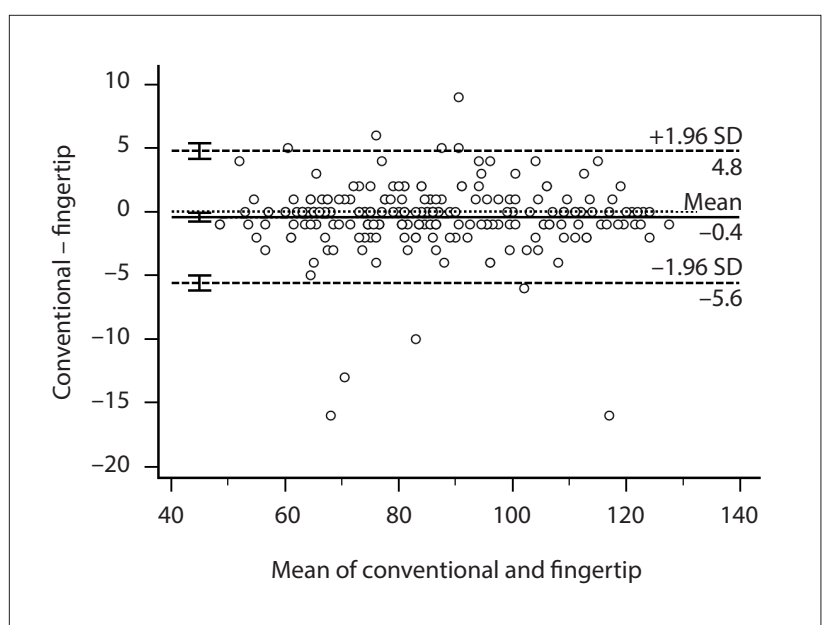

Fig. 4. Bland-Altman plot for pulse rate measurements $(b p m)$. $(S D=$ standard deviation.)

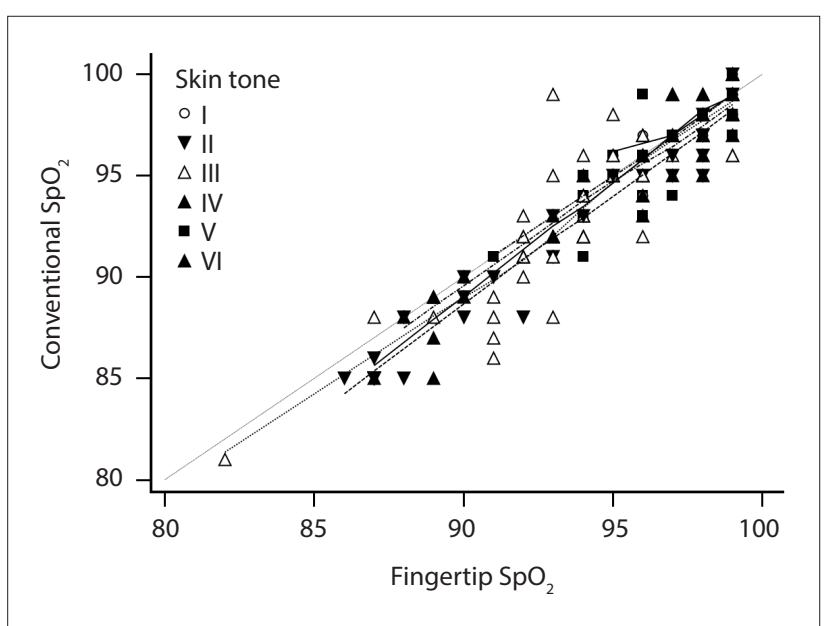

Fig. 5. Oximeter $\mathrm{SpO}_{2}$ (\%) correlation by skin tone classification. For definitions of skin tones $I-V I$, see Table 2. ( $\mathrm{SpO}_{2}=$ peripheral oxygen saturation.)

progressively deteriorates below an $\mathrm{SaO}_{2}$ of $90 \%$. There is currently insufficient published evidence that portable fingertip pulse oximeters are as accurate as standard pulse oximeters in estimating arterial oxygen saturation in a clinical setting, as opposed to the controlled environment of a laboratory. Various unpublished accuracy studies have been performed on individual manufacturers' fingertip pulse oximeters. ${ }^{[28]}$ They are not peer reviewed and usually recruit healthy adult volunteers under laboratory-controlled hypoxic conditions.

Most fingertip pulse oximeters marketed to consumers for nonmedical use do not undergo stringent testing as laid out by the FDA and other regulatory bodies. ${ }^{[15]}$ Lipnick et al. ${ }^{[15]}$ found that only two of six such commonly used devices met ISO and FDA standards. Of these, one was the fingertip pulse oximeter included in this study. All oximeters demonstrated deteriorating accuracy at lower arterial oxygen saturations. Some of the important limitations of the study identified by the authors were the ideal conditions under which the pulse oximeters were tested (motionless hands of healthy volunteers with good perfusion in a laboratory utilising controlled hypoxic gas mixtures). They warned about extrapolating these data to the clinical setting, where multiple factors and patient comorbidities could potentially affect the pulse oximeters' accuracy. The majority of the volunteers had lighter skin tones, and were therefore not representative of a population like that of SA.

A study involving 55 dental patients in Brazil found no statistically significant difference between a portable fingertip oximeter and hospital pulse oximeters. ${ }^{[29]}$ The non-invasive $\mathrm{SpO}_{2}$ of each device was measured by simultaneous application of the fingertip probe at six time intervals during the dental procedures. Although this study was conducted in a clinical setting, it included insufficient comparisons for a Bland-Altman analysis, and the Pearson correlation coefficient reported is not an appropriate method for equipment comparison. ${ }^{[20]}$ An additional 2015 clinical study examined the agreement between a portable fingertip pulse oximeter (Maxtec MD3OO C2) and $\mathrm{SaO}_{2}$ measured by a laboratory blood gas analyser. ${ }^{[30]}$ Patients in a pulmonary and renal intermediate care unit were recruited by convenience sampling when a 'therapeutically prescribed' arterial blood gas value was required. The $\mathrm{SaO}_{2}$ was then compared with $\mathrm{SpO}_{2}$ derived by the test fingertip pulse oximeter, which was applied within 3 minutes of the blood gas sample. The authors noted an unacceptable difference in bias and precision values, especially when the $\mathrm{SaO}_{2}$ was $\leq 93 \%$. There were some significant methodological flaws, however, including the study being underpowered $(N=32)$, and exclusion criteria not consistent with previous studies that highlighted common causes of error in saturation measurement. The pulse oximeter was not always applied simultaneously with arterial sampling, and in 21 of the 32 samples the delay of up to 3 minutes in obtaining the $\mathrm{SpO}_{2}$ may have resulted in measurement of a different value to that obtained by the arterial blood gas sample.

Our study adds value to the existing literature in that it evaluates the performance of fingertip pulse oximeters in the clinical setting, among patients with varying American Society of Anesthesiologists (ASA) status and more heterogeneous skin tones. Another merit of the study is that data measurements were obtained across a range of perioperative clinical settings (ICU, recovery room, clinic, theatre).

One of the goals of the COMFORT trial is to create a methodology that can be easily and cheaply reproduced in resource-constrained healthcare environments in other countries to test the clinical agreement of various inexpensive, portable fingertip pulse oximeters with the existing conventional pulse oximeters in these institutions. If there is a predominant/homogeneous skin tone in a particular setting, these data may be omitted.

\section{Study limitations}

An inherent limitation of this pragmatic study is the skewed data obtained when measuring $\mathrm{SpO}_{2}$ in the clinical setting. Most samples $(91.4 \%)$ were in the $\mathrm{SpO}_{2}$ range $\geq 90 \%$, and while the data do not 
address the questions regarding accuracy at $\mathrm{SpO}_{2}<80 \%$, they may be a more accurate reflection of $\mathrm{SpO}_{2}$ encountered in daily clinical practice. A further minor source of bias was that the fingertip pulse oximeter only displayed a two-digit reading for $\mathrm{SpO}_{2}$ (omitting 100\%). As there is no clinically meaningful difference between saturations of $99 \%$ and $100 \%$, we elected to include these differences, which did not affect the primary outcome. However, the true value of an accurate pulse oximeter lies in its ability to correctly identify a hypoxaemic patient who would require prompt intervention. Clinicians could infer from this study that fingertip pulse oximeter readings $\geq 93 \%$ are reassuring while those $<93 \%$ require further investigation and should prompt treatment if the patient has reversible causes of mild or profound hypoxaemia. Baseline saturations in the context of the patient's underlying respiratory condition should of course be considered when deciding whether treatment is required; the clinician is reminded to 'treat the patient, not the monitor'.

While the study does include a range of skin tones more representative of SA population demographics than previous studies, each subgroup is underpowered to detect a statistically significant difference. The authors noted no clinically significant difference in pulse rates or $\mathrm{SpO}_{2}$ values between lighter- and darker-pigmented patients, and both statistical and graphical analysis of the trends in measurement by skin tone grouping showed no difference between the devices. Lack of more patient-specific data (e.g. age, sex, ASA status) makes more detailed analysis of the data impossible. The difference in averaging time of the two devices may have adversely affected the data in situations where saturations were changing rapidly, as might be encountered in a setting such as the recovery room.

\section{Conclusions}

This pragmatic study demonstrated that a fingertip pulse oximeter was accurate (within $3 \% \mathrm{SpO}_{2}$ ) in perioperative patients with normal oxygenation $\left(\mathrm{SpO}_{2} \geq 93 \%\right)$ compared with a bedside pulse oximeter. As in previous studies, accuracy deteriorated with progressive hypoxaemia. A measurement of $<93 \%$ on the portable device is cause for concern, and should prompt further investigation and management of hypoxia if necessary. Pulse rates measured by the portable devices were of clinically acceptable accuracy. We found that darker skin pigmentation showed no trend to an effect on the accuracy of either measurement. Future studies are required to investigate the agreement between these two devices at lower oxygen saturation levels, and studies should be specifically powered to detect differences in accuracy between varying skin tones.

Declaration. This study was performed in partial completion of the degree MMed (Anaesthesiology) at the University of Cape Town (RNS).

Acknowledgements. The authors wish to acknowledge the staff and patients of Groote Schuur Hospital, the experienced senior researchers in the Department of Anaesthesia and Perioperative Medicine, and the Western Cape Provincial Government Department of Health for their selfless assistance.

Author contributions. RH: study conception, initial design, pilot study, statistical analysis, manuscript review, editing and supervision; RNS: study protocol, literature review, data collection, manuscript writing and review.
Funding. This study was supported by departmental funds. The CMS50D portable fingertip pulse oximeter used in the study was purchased by the authors.

Conflicts of interest. None.

1. Merry AF, Eichhorn JH, Wilson IH. Extending the WHO 'Safe Surgery Saves Lives' project through global oximetry. Anaesthesia 2009;64(10):1045-1048. https://doi.org/10.1111/j.13652044.2009.06104.x

2. Bettings P, Diedericks J, Fourie P, et al. South African Society of Anaesthesiologists Practice Guidelines: 2012 Revision. S Afr J Anaesth Analg 2013;19(1):S1-42. https://doi.org/10.1080/2220
(a) Guidelines: 2012 Revision

1173.2013 .10872899
3. Mitchell J. Recommendations for standards of monitoring during anaesthesia and recovery. Mitchell J. Recommendations for standards of monitoring during anaesthesia
Anaesthesia 2001;56(5):488-488. https://doi.org/10.1046/j.1365-2044.2001.02047-7.x

4. Merry A, Cooper J, Soyannwo O, Wilson I, Eichhorn J. International Standards for a Safe Practice of Anesthesia 2010. Can J Anaesth 2010;57(11):1027-1034. https://doi.org/10.1007/s12630-010-9381-6

5. World Alliance for Patient Safety. WHO Surgical Safety Checklist and Implementation Manual. World Health Organization, 2008. http://www.who.int/patientsafety/safesurgery/ss_checklist/en/ (accessed 21 June 2017).

6. Haynes AB, Weiser TG, Berry WR, et al. A surgical safety checklist to reduce morbidity and mortality in a global population. N Engl J Med 2009;360:491-499. https://doi.org/10.1056/NEJMsa0810119

7. Food and Drug Administration. Pulse oximeters - premarket notification submissions [510(k)s] Guidance for industry and Food and Drug Administration staff. 4 March 2013. https://www.fda.gov/ RegulatoryInformation/Guidances/ucm341718 (accessed 12 February 2019).

8. Moller JT, Pedersen T, Rasmussen LS, et al. Randomized evaluation of pulse oximetry in 20,802 Moller JT, Pedersen T, Rasmussen LS, et al. Randomized evaluation of pulse oximetry in 20,802
patients: I. Design, demography, pulse oximetry failure rate, and overall complication rate. patients: I. Design, demography, pulse oximetry failure rate, and overall complication
Anesthesiology 1993;78(3):436-444. https://doi.org/10.1097\%2F00000542-199303000-00006

9. Moller JT, Johannessen NW, Espersen K, et al. Randomized evaluation of pulse oximetry in 20,802 9. Moller JT, Johannessen NW, Espersen K, et al. Randomized evaluation of pulse oximetry in 20,802
patients: II. Perioperative events and postoperative complications. Anesthesiology 1993;78(3):445453. https://doi.org/10.1097/00000542-199303000-00007

10. Sinex J. Pulse oximetry: Principles and limitations. Am J Emerg Med 1999;17(1):59-66. https://doi. org/10.1016/S0735-6757(99)90019-0

11. Jubran A. Pulse oximetry. Crit Care 2015;19:272. https://doi.org/10.1186/s13054-015-0984-8

2. Chitilian H, Kaczka D, Vidal Melo M. Respiratory monitoring. In: Miller R, ed. Miller's Anesthesia. 8th ed. Philadelphia: Elsevier Saunders, 2015:1541-1579.

13. Jensen LA, Onyskiw JE, Prasad NGN. Meta-analysis of arterial oxygen saturation monitoring by pulse oximetry in adults. Heart Lung 1998;27(6):387-408. https://doi.org/10.1016/S01479563(98)90086-3

14. Bickler P, Feiner J, Severinghaus J. Effects of skin pigmentation on pulse oximeter accuracy at low saturation. Anesthesiology 2005;102(4):715-719. https://doi.org/10.1097/00000542-20050400000004

15. Lipnick M, Feiner J, Au P, Bernstein M, Bickler P. The accuracy of 6 inexpensive pulse oximeters not cleared by the Food and Drug Administration: The possible global public health implications. Anesth Analg 2016;123(2):338-345. https://doi.org/10.1213/ANE.0000000000001300

16. Lu MJ, Zhong WH, Liu YX, Miao HZ, Li YC, Ji MH. Sample size for assessing agreement between two methods of measurement by Bland-Altman method. Int J Biostat 2016;12(2). https://doi org/10.1515/ijb-2015-0039

7. Fitzpatrick T. Soleil et peau. J Med Esthet 1975;2:33-34.

8. Fitzpatrick T. The validity and practicality of sun-reactive skin types I through VI. Arch Dermatol 1988;124(6):869-871. https://doi.org/10.1001/archderm.1988.01670060015008

9. Sanyal S, Nundy KK. Algorithms for monitoring heart rate and respiratory rate from the video of a user's face. IEEE J Transl Eng Health Med 2018;6(May). https://doi.org/10.1109/jtehm.2018.2818687

20. Bland J, Altman D. Statistical methods for assessing agreement between two methods of clinica measurement. Lancet 1986:327(8476):307-310. https://doi.org/10.1016/S0140-6736(86)90837-8

1. Bland JM, Altm DG. Compang methods of mesurement: Why ploting difference again Bland JM, Altman DG. Comparing methods of measurement: Why plotting difference against standard method is mis

Measurement in medicine: The analysis of method comparison studies. J R Stat Soc Ser D Statistician 1983;32(3):307-317. https://doi.org/10.2307/2987937

23. Bland JM, Altman DG. Measuring agreement in method comparison studies. Stat Methods Med Res 1999;8(2):135-160. https://doi.org/10.1177/096228029900800204

24. Jubran A. Advances in respiratory monitoring during mechanical ventilation. Ches 1999;116(5):1416-1425. https://doi.org/10.1378/chest.116.5.1416

25. Webb R, Ralston A RW. Potential errors in pulse oximetry: II. Effects of changes in saturation and signal quality. Anaesthesia 1991;46(3):207-212. https://doi.org/10.1111/j.1365-2044.1991.tb09411.x 6. Taylor M, Whitwam J. The accuracy of pulse oximeters. Anaesthesia 2007;43(3):229-232. https://doi. org/10.1111/j.1365-2044.1988.tb05549.x

27. Nickerson B, Sarkisian C, Tremper K. Bias and precision of pulse oximeters and arterial oximeters. Chest 1988;93(3):515-517. https://doi.org/10.1378/chest.93.3.515

28. Colechin E, Bousfield D, Reay C, Sims A. Market review: Pulse oximeters in primary and prehospital care. 2010;(March). Originally available from Centre for Evidence-based Purchasing (CEP); web page decommissioned. Now available from http://openairway.org/colechin-et-al-2010-marketpage decommissioned. Now available from http://openairway.org/colechin-et-al-20
review-pulse-oximeters-in-primary-and-prehospital-care/ (accessed 12 February 2019).

29. Takeshita WM, Iwaki LCV, Pupim D, Filho L. Evaluation of accuracy of portable fingertip pulse oximeter, as compared to that of a hospital oximeter with digital sensor. Indian J Dent Res oximeter, as compared to that of a hospital oximeter with
2013;24(5):542-546. https://doi.org/10.4103/0970-9290.123362

30. Jones M, Olorvida E, Monger K, et al. How well do inexpensive, portable pulse oximeter values agree with arterial oxygenation saturation in acutely ill patients? Medsurg Nurs 2015;24(6):391 396. https://www.thefreelibrary.com/How well do inexpensive, portable pulse oximeter values agree with...-a0439362279 (accessed 12 February 2019).

Accepted 13 November 2018. 